\title{
BMJ Open Effect of iron supplementation on psychomotor development of non-anaemic, exclusively or predominantly breastfed infants: a randomised, controlled trial
}

\author{
Anna Chmielewska, ${ }^{1}$ Grzegorz Chmielewski, ${ }^{2}$ Magnus Domellöf, ${ }^{3}$ \\ Zbigniew Lewandowski, ${ }^{4}$ Hania Szajewska ${ }^{1}$
}

To cite: Chmielewska $A$, Chmielewski G, Domellöf M, et al. Effect of iron supplementation on psychomotor development of non-anaemic, exclusively or predominantly breastfed infants: a randomised, controlled trial. BMJ Open 2015;5: 009441.

doi:10.1136/bmjopen-2015009441

- Prepublication history for this paper is available online. To view these files please visit the journal online (http://dx.doi.org/10.1136/ bmjopen-2015-009441)

Received 19 July 2015 Revised 10 September 2015 Accepted 30 September 2015

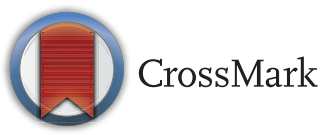

${ }^{1}$ Department of Paediatrics, Medical University of Warsaw, Warsaw, Poland ${ }^{2}$ Second Department of Obstetrics and Gynaecology, Medical University of Warsaw, Warsaw, Poland ${ }^{3}$ Department of Clinical Sciences, Pediatrics, Umea University, Umea, Sweden

${ }^{4}$ Department of

Epidemiology, Medical

University of Warsaw,

Warsaw, Poland

Correspondence to Dr Anna Chmielewska; aachmielewska@gmail.com

\section{ABSTRACT}

Introduction: Uncertainty exists regarding the effects of iron supplementation during infancy on neurodevelopmental outcomes in the absence of anaemia. The aim of the study is to establish whether psychomotor and mental development is influenced by early iron supplementation in healthy, non-anaemic, exclusively or predominantly breastfed infants.

Methods and analysis: Healthy term infants will be recruited. If exclusively or predominantly breast fed (>50\% of daily feedings) and not anaemic at 4 months, they will be randomised to receive either iron pyrophosphate (approximately $1 \mathrm{mg} / \mathrm{kg}$ ) or placebo daily until 9 months of age. The primary outcome measure is neurodevelopment assessed with the Bayley Scales of Infant and Toddler Development (Bayley-III) at 12 months, and repeated at 24 and 36 months of age. Haematological parameters of iron metabolism also will be measured.

Ethics and dissemination: The Bioethics Committee of the Medical University of Warsaw approved the study protocol before recruitment started. Study results will be submitted to peer-reviewed journals in the fields of paediatrics and nutrition, and presented at relevant conferences.

Trial registration number: NCT02242188.

\section{INTRODUCTION}

Iron is a nutrient of essential importance to the human organism. It takes part in energy production, oxygen transportation and DNA synthesis, and is indispensable for the development of the central nervous system. Iron is required for the myelination and production of neurotransmitters. It has been well documented that iron deficiency anaemia (IDA), that is, decreased concentration of haemoglobin $(\mathrm{Hb})$ and depleted iron stores, is associated with impaired child development. ${ }^{1-3}$ If the diagnosis of IDA is delayed, the deficits

\section{Strengths and limitations of this study}

- A precise clinical question has been posed to fill a gap in knowledge: Does iron supplementation in healthy, non-anaemic, breastfed infants influence their psychomotor and mental development?

- The study design (randomised controlled trial) is the methodology of choice to assess the effectiveness of such an intervention.

- The assessment of development at several time points up to 36 months of age will allow detection of possible long-term effects of iron supplementation.

- A longer follow-up period would be valuable, but probably will not be feasible due to attrition and cost.

may be irreversible. ${ }^{4}$ A recent Cochrane review stated that iron treatment may improve developmental outcomes in young children with IDA, but the existing evidence is scarce and there is an urgent need for trials assessing long-term effect of this therapy. ${ }^{5}$ Iron deficiency (ID) is the most common single nutrient deficiency and may affect up to $20 \%$ of children of 1-3 years of age in Europe. ${ }^{6}$ ID has the potential to negatively influence psychomotor development. However, a causal relationship is not as clear as that for IDA. ${ }^{7-9}$ Exclusive breast feeding increases the risk of ID, but controversies exist about whether iron supplementation in this population should be recommended. ${ }^{6} 10$

Previous studies have suggested that iron supplementation in healthy infants may enhance psychomotor development. A meta-analysis carried out by our group (Szajewska et $a l^{11}$ ) evaluated the effects of iron supplementation in non-anaemic 
pregnant women and in non-anaemic healthy children aged $<3$ years on the mental performance and psychomotor development of the children. Seven randomised controlled trials (RCTs) were identified, five of which referred to supplementation during infancy. ${ }^{12-16}$ Although the included trials were of relatively good methodological quality (all were blinded and most of these were randomised, with proper allocation concealment), the major limitation was incomplete outcome data ( $>20 \%$ lost to follow-up) and risk of selective reporting (study protocols were not available). The pooled results of three RCTs $(\mathrm{n}=561)$ showed significant improvement on the Psychomotor Development Index (PDI) of the Bayley Scales of Infant Development (BSID) at approximately 12 months of age in the ironsupplemented group compared with the control group (mean difference: 4.21 ; $95 \%$ CI 2.31 to 6.12 ). No significant effect of iron supplementation on the Mental Development Index (MDI) or behaviour was found. A follow-up study of children aged 9 years who participated in a randomised trial during infancy (daily supplementation of iron, zinc, iron and zinc, or placebo at 46 months of age for 6 months; $92 \%$ of participants available for follow-up evaluation) has been recently published. ${ }^{15}$ Cognitive and school performance did not differ significantly between the four groups. ${ }^{17}$

Another recently published systematic review aimed to assess effectiveness of oral iron treatment to improve the developmental and haematological outcomes of preschool children (1-5 years) with non-anaemic ID (normal $\mathrm{Hb}$ concentration and depleted iron stores). ${ }^{18}$ Two randomised trials were identified $(n=69)$, both with moderate risk of bias due to insufficient information on allocation concealment and open-label intervention in one of the trials. One trial showed a statistically significant difference in the post-treatment MDI score among children who received oral iron therapy compared with no therapy (mean difference: $6.3,95 \%$ CI 1.5 to 11.0 ). Authors concluded that the evidence was insufficient to recommend oral iron therapy to school-aged children with non-anaemic ID. Adequately powered, randomised trials are needed in children with non-anaemic ID.

Given that ID is a common problem in young children, measures to prevent it are being taken. According to the Committee of Nutrition of the European Society for Paediatric Gastroenterology, Hepatology and Nutrition (ESPGHAN CoN), this should be achieved by delayed umbilical cord clamping, the use of ironfortified formulas when formula feeding is needed, the postponement of the introduction of whole cow milk as the main drink until the end of the first year of life, and the promotion of consumption of complementary foods rich in iron. ${ }^{6}$ Furthermore, the ESPGHAN CoN concluded that there is no convincing evidence that iron supplements should be provided to normal birth weight, exclusively breastfed infants during the first 6 months of life in populations with a low prevalence of IDA among 6-month-old infants. In contrast, the American Academy of Pediatrics (AAP) recommends iron supplementation $(1 \mathrm{mg} / \mathrm{kg} /$ day $)$ in exclusively breastfed infants beginning at 4 months of age that should be continued until iron from complementary foods is available. ${ }^{10}$ As the level of iron intake is uncertain in partially breastfed infants, the AAP recommends that those who receive more than one-half of their daily feedings as human milk also should be supplemented with $1 \mathrm{mg} / \mathrm{kg} /$ day of iron beginning at 4 months. ${ }^{10}$

This considerable difference between European and US guidelines is based on different interpretations of the scarce available evidence. The only study that has previously addressed this issue was performed by, among others, a member of our group (MD). In that study, 101 Swedish and 131 Honduran, non-anaemic, breastfed infants were randomised into three groups: placebo from 4 to 9 months, iron supplements $(1 \mathrm{mg} / \mathrm{kg} /$ day $)$ from 4 to 9 months, or placebo from 4 to 6 months and iron supplements from 6 to 9 months of age. ${ }^{19}$ The study showed that iron supplements effectively decreased the risk of IDA at 9 months in Honduran infants, but not in the Swedish infants, who already had a low prevalence of IDA at 9 months $(<3 \%)$. However, in the Swedish infants who received iron supplements, a negative effect was observed on growth. ${ }^{20}$ Unfortunately, these infants were not followed up for a longer period than 9 months, and neurodevelopmental outcomes were not assessed.

There is, therefore, a clear need for interventional studies of good methodological quality to evaluate the role of iron supplementation in non-anaemic infants on their mental and psychomotor development, as well as on possible adverse effects on growth and infections (A Chmielewska, P Dziechciarz, D Gieruszczak-Białek, et al. Personal communication, 2015). ${ }^{6}$

Iron preparations in the form of syrup or drops, which are usually prescribed for the treatment of anaemia in children, have an unpleasant taste, may stain the teeth, and cause adverse effects mostly related to the gastrointestinal tract. Additionally, these preparations need careful measuring to obtain the desired dose. ${ }^{21}$ Micronised ferric pyrophosphate is a novel form of microencapsulated iron, which can be packaged in easy-to-use sachets and added to milk or weaning foods. It has been generally recognised as safe as a nutrient supplement in food. ${ }^{22}$ Ferric pyrophosphate, marketed as SunActive Fe, has been shown to be effective in infants with anaemia. ${ }^{23}$ Its use was less often related to episodes of diarrhoea, vomiting and teeth staining compared with ferrous glycine sulfate drops. ${ }^{23}$ The easy-to-use form of the powder, which can be added to liquids or foods without changing the taste, makes longterm supplementation easier to pursue. ${ }^{21}$

\section{Trial objectives and hypotheses}

The main objective of this trial is to assess the effectiveness of low-dose iron $(1 \mathrm{mg} / \mathrm{kg} /$ day $)$ supplementation in healthy, term, breastfed infants from 4 to 9 months of age with regard to optimising their developmental 
outcomes measured at 12, 24 and 36 months of age. The hypothesis being tested is that psychomotor and mental development will be superior in ironsupplemented children.

Secondary objectives are to assess the effects of iron supplementation on infant growth (weight, length, head circumference) and laboratory parameters of iron status ( $\mathrm{Hb}$, mean corpuscular volume (MCV), haematocrit, serum ferritin (s-Ferritin), reticulocyte $\mathrm{Hb}$, hepcidin and soluble transferrin receptor concentration (sTfR)). Behaviour at 36 months will also be assessed.

\section{METHODS AND ANALYSIS}

The trial is registered at http://www.clinicaltrials.gov (NCT02242188). Any important modifications in the protocol will be entered there.

\section{Study design}

Pragmatic, blinded, parallel-group, superior, randomised, placebo-controlled trial.

\section{Setting and participants}

Healthy singleton infants will be considered for inclusion before completion of 4 months of age. Parents will be approached either shortly after birth at the obstetrics department (a tertiary care clinical hospital for women: Department of Obstetrics and Gyneacology, Medical University of Warsaw, Poland) or during well-baby visits at general practitioners' offices located within the community of Warsaw. Those who are eligible will be invited to participate in the study. Parents considering participation will receive oral and written information on the study. A researcher will contact the caregivers by telephone at approximately 3.5 months of age to check for the eligibility criteria again. Those exclusively breast fed or predominantly breast fed, that is, receiving breast milk for over $50 \%$ of feedings at the age of 4 months, will be considered for inclusion and invited to the study site. After caregivers provide informed consent, an infant will be included and blood will be obtained. Those without anaemia, defined as $\mathrm{Hb}<105 \mathrm{~g} / \mathrm{L}$ $(<10.5 \mathrm{~g} / \mathrm{dL}),{ }^{5}$ will be randomised to receive either the iron supplement or placebo from the age of 4 completed months until 9 months of age. Apart from recruitment, all study procedures will be pursued in the Department of Paediatrics, Medical University of Warsaw, Poland.

\section{Inclusion criteria}

To be included in the study, a child must be a healthy singleton infant of age 1 day to 4 months, born at term (37-42 weeks of gestation) with a normal birth weight of $>2500 \mathrm{~g}$. If approached shortly after delivery, the mother of a child must express the intention to breast feed. If the child is recruited at an older age, an infant must be breast fed either exclusively or predominantly $(>50 \%$ feedings). A caregiver must provide written informed consent. This will be obtained by one of the two physicians involved in the study.

\section{Exclusion criteria}

The following exclusion criteria will be applied: preterm delivery ( $<37$ weeks of gestation), birth weight $<2500 \mathrm{~g}$, multiple pregnancy, major illness or congenital anomaly, being $<50 \%$ breast fed at the time of inclusion, food allergy, anaemia $(\mathrm{Hb}<105 \mathrm{~g} / \mathrm{L}(10.5 \mathrm{~g} / \mathrm{dL}))$ at inclusion, lack of informed consent and difficult communication with caregivers.

\section{Randomisation criteria}

The participants will be randomised at the age of 4 completed months, after rechecking the inclusion and exclusion criteria, under the condition of being exclusively breast fed or receiving breast milk for at least $50 \%$ of their daily feeds at the time of randomisation.

\section{Interventions}

Infants in the experimental group will receive the powder preparation of iron pyrophosphate and maltodextrin (Actiferol, SunActive Fe, Sequoia, Poland) in a single daily dose of approximately $1 \mathrm{mg} / \mathrm{kg}$ of body weight from 4 to 9 months of age (dose in line with the AAP recommendations). Three doses will be used: $7 \mathrm{mg}$ for infants up to $7 \mathrm{~kg}$ of body weight, $10 \mathrm{mg}$ for infants from 7 to $10 \mathrm{~kg}$ of body weight, and $15 \mathrm{mg}$ for those exceeding the weight of $10 \mathrm{~kg}$. Caregivers will be instructed to administer the daily dose at the same time of a day after mixing the content of the sachet with a little amount of breastmilk or milk formula. Infants in the placebo group will receive maltodextrin alone. Maltodextrin is an almost flavourless, easily digestable polysaccharide commonly used as a food additive.

\section{Allocation concealment and blinding}

A computer-generated randomisation list prepared by a person unrelated to the trial will be used to allocate participants to the study groups (A or B) in blocks of 10 . Stratification by infant gender will be applied. Consecutive randomisation numbers (each number assigned to intervention or placebo in a blinded way, as described below) will be given to participants at enrolment. This procedure will be performed by one of the two physicians involved. The study products will be delivered to the centre in boxes labelled with the letters $\mathrm{A}$ and $\mathrm{B}$ (meaning of A and B will be blinded, information deposited in a sealed envelope in a safe at the administrative part of the department). The boxes will also carry the information on the specific dose $(7,10$ or $15 \mathrm{mg})$. Subsequently, the letters A and B will be removed from the boxes by an independent person unrelated to the study planning and conduct, and replaced with numeric codes corresponding to the randomisation numbers, for example, 001-7, 001-10, 001-15, 002-7, 002-10, 002-15 mg, etc. Sachets containing the study product will be packed in small packages of 30 pieces each. 
Neither the collective packages nor the sachets will carry any labelling and all the unused sachets will always be returned by the parents before switching to a higher dose. The active product and placebo will be packed in identical sachets, and the content will look and taste the same. Researchers, caregivers, outcome assessors and a person responsible for the statistical analysis will be blinded to the intervention until a statistical report for the 12 months' developmental assessment is available. After that, both the caregivers and main outcome assessor will remain blinded until the completion of the study. The information on intervention assignment will be stored in a sealed envelope in a safe in the administrative part of the department.

The personal information about potential and enrolled participants will be stored in a locker within the study site, accessible to the involved researchers only.

\section{Compliance}

The caregivers will be asked to bring the remaining study product to the study site each time they bring the infant for a check-up visit during the intervention, that is, at 6 and 9 months of age. Compliance with the study protocol will be checked by counting the number of sachets left unused. Those participants receiving less than $75 \%$ of the recommended doses will be considered non-compliant.

\section{Primary outcome}

Psychomotor development at the age of 12 months will be the primary outcome measure. Additional assessments will be performed at 24 and 36 months. The Bayley Scales of Infant and Toddler Development (Bayley-III or BSID-III) will be used. ${ }^{24}$ Fine and gross motor, cognitive, language, and social-emotional development scores will be measured with the Bayley-III (in the previous version of this tool (BSID-II) these components of assessment were presented as PDI and MDI). A psychologist qualified and experienced in assessment with use of Bayley-III will perform the test within 1 month of the time point when a child reaches 12, 24 and 36 months of age. Psychomotor development as the main outcome of the study will be adjusted for gestational age, parental education and socioeconomic status.

\section{Secondary outcomes}

Behaviour

At 3 years, additional screening for behavioural and emotional problems will be performed with use of Child Behaviour Checklist (CBCL), a version for preschool children. ${ }^{25}$ This will be applied by the psychologist performing the psychomotor development assessment.

\section{Laboratory tests}

Haematological status will be assessed at 4, 12 and 24 months. Samples will be analysed for $\mathrm{Hb}$ concentration, MCV, reticulocyte $\mathrm{Hb}$ concentration, s-Ferritin concentration and $\mathrm{C}$ reactive protein. Novel parameters of iron status, such as hepcidin concentration, and sTfR will also be measured. Hepcidin is a recently characterised oligopeptide that has emerged as the master regulator of iron metabolism. ${ }^{26}{ }^{27}$ It has been shown to be closely associated with iron status and iron intakes in infants. ${ }^{28}$ Further evaluation of hepcidin and sTfR in children has been listed among the future research directions by the ESPGHAN Committee on Nutrition. ${ }^{6}$ Hepcidin will be analysed by ELISA (BioTek ELx800) and sTfR with the use of immunoturbidimetric method (COBAS c501, Roche). Blood samples will be taken by paediatric nurses and analysed at the study site.

IDA will be defined as $\mathrm{Hb}$ concentration $<105 \mathrm{~g} / \mathrm{L}$ (ie, $<10.5 \mathrm{~g} / \mathrm{dL}$ ) and s-Ferritin concentration of $<45 \mathrm{pml} / \mathrm{L}$ (ie, $<20 \mu \mathrm{g} / \mathrm{L}$ ) at 4 months or $<27 \mathrm{pml} / \mathrm{L}$ (ie, $<12 \mu \mathrm{g} / \mathrm{L}$ ) for 12 and 24 months. ${ }^{6}$ Non-anaemic ID will be defined as $\mathrm{Hb}$ concentration $>105 \mathrm{~g} / \mathrm{L}$ and s-Ferritin concentration $<45 \mathrm{pml} / \mathrm{L}$ at 4 months or $<27 \mathrm{pml} / \mathrm{L}$ for subsequent measurements. ${ }^{6}$ Children diagnosed with anaemia will be offered clinical evaluation and treatment within the department or referred to a paediatrician.

\section{Growth}

Growth parameters (weight, length/height and head circumference) will be recorded during the follow-up visits at 4, 6, 9, 12, 24 and 36 months of age. Measurements will be performed by paediatric nurses in a standardised way with use of periodically calibrated scales and measuring boards, as per the WHO recommendations. ${ }^{29}$

\section{Dietary iron intake}

Dietary assessment in terms of iron intake will be performed at 9 months by means of a 3-day, prospective food diary, which will be prepared on a standardised form by the caregiver(s) and delivered to a health provider at the 9-month follow-up visit. Iron intake will be calculated with use of a DIETA 5.D software (V.2015) ${ }^{30}$

\section{Adverse events}

Parents will be asked to fill out a form of possible adverse events of the intervention daily. The symptoms listed in the form will include diarrhoea, vomiting, constipation, discolouration of the stool, fever and respiratory tract infections. The forms will be collected at each check-up visit during the intervention.

Data collected during the study will be stored safely in a locker accessible only to the researchers involved in the study. Data will be transferred from the paper case report forms to the electronic database. Double data entry will be applied. The final data set will be available to the authors of this protocol only.

\section{Timeframes of activities during the study}

Recruitment for the trial has started in August 2015 and the whole study, including the 36-month follow-up, will last until mid-2018. The time points of all participant-related actions to be taken during the study period are presented in table 1 . 
Table 1 Timetable of activities planned during the course of the study directly related to participants

\begin{tabular}{|c|c|c|c|c|c|c|c|c|c|c|c|c|}
\hline \multirow[b]{2}{*}{ Activity } & \multicolumn{12}{|c|}{ Age (months) } \\
\hline & 1 & 4 & 5 & 6 & 7 & 8 & 9 & 10 & 11 & 12 & 24 & 36 \\
\hline Enrolment & + & & & & & & & & & & & \\
\hline Randomisation & & + & & & & & & & & & & \\
\hline Intervention & & & + & + & + & + & + & & & & & \\
\hline Dietary assessment & & & & & & & + & & & & & \\
\hline Anthropometry & & + & & + & & & + & & & + & + & + \\
\hline Blood withdrawals & & + & & & & & & & & + & + & \\
\hline $\begin{array}{l}\text { Neurodevelopmental assessment } \\
\text { Behaviour }\end{array}$ & & & & & & & & & & + & + & $\begin{array}{l}+ \\
+\end{array}$ \\
\hline
\end{tabular}

\section{Retention of participants in the study}

Retaining participants in a clinical trial of long-term duration represents a substantial difficulty for research teams. To make the obtained results the most credible, a high percentage of participants should complete the study and their data should ideally be available for the assessment of main outcomes. ${ }^{31}$ The current study is a trial with a relatively long intervention period and a long follow-up. To avoid high attrition rate, actions recommended in the literature are planned. ${ }^{32}$ The caregivers of participating children will be free to contact a researcher (paediatrician) or a nurse by telephone or email at any time. The researchers and study nurse will contact parents by telephone during the intervention period to ensure that the infant receives the supplement. Visits will be scheduled by telephone 2 weeks in advance, and the parents will receive a reminder text message on the day before the scheduled visit. Transportation cost refund will be offered.

\section{Data monitoring}

Data monitoring committee has not been established since the intervention within the trial (iron $1 \mathrm{mg} / \mathrm{kg}$ per day) does not differ from standard of care in infants from risk groups. ${ }^{6}$ The profile of potential side effects is also known.

\section{Power calculation}

The sample size was calculated for the main outcome of fine and gross motor, cognitive, language, and social-emotional development scores to be measured using the Bayley-III test at the age of 12 months. ${ }^{24}$ In the previous editions of the test, these elements of the assessment were combined into the PDI and the MDI. To detect a difference of five points in the PDI between the study groups with a power of $80 \%$ and $\alpha=0.05$, a sample of 91 infants is needed in each study group. This sample size is based on the assumption that the SD would be 12 points for the PDI in each study group. To account for $20 \%$ of loss to follow-up, we aim to recruit a total of 220 infants for this study.

Achieving the target sample size might be challenging within the planned period. If the recruitment pace is considered to be too slow, all efforts will be made to increase the number of parents approached mainly by involving additional paediatric practices.

\section{Statistical analysis}

The SAS System (SAS System V.9.4, SAS Institute Inc, Cary, North Carolina, USA, 2013) will be used for calculations: Power, NPAR1WAY, TTEST and MIXED Procedure. Results will be analysed on an intention-to-treat basis. Per-protocol analysis will be applied to compliers only ( $>75 \%$ of doses taken). The experimental and control groups will be compared in terms of developmental scores by means of the Student $\mathrm{t}$ test or the Mann-Whitney test. Repeated-measures analysis of variance will be performed for developmental scores at 12, 24 and 36 months. For categorical variables, Fisher's exact test will be used. In exploratory analysis, two factors will be taken into account: gender and birth weight (2500-3000 vs >3000 g). Psychomotor development as the main outcome of the study will be adjusted for gestational age, parental education and socioeconomic status. A two-tailed $p$ value of the test statistics $<0.05$ will be considered to be significant.

\section{Practical importance of the project}

Iron supplementation in infants at risk of iron depletion is an established practice and one of the main objectives of this project is to optimise neurodevelopment by means of preventing non-anaemic ID and anaemia. Exclusive breast feeding increases the risk of ID, but controversies exist about whether iron supplementation in this population should be recommended. The results of our project may shed light on these uncertainties and will contribute to optimising child healthcare. If repeated by other research teams, these results may substantially influence early feeding recommendations and practices.

\section{ETHICS AND DISSEMINATION}

The Bioethical Committee of the Medical University of Warsaw issued the study approval before recruitment started. Any important modifications in the protocol will be communicated to the Committee. The full protocol will be available freely due open access publication. The 
findings of this RCT will be submitted to a peer-reviewed journal (paediatric, nutrition or gastroenterology). Abstracts will be submitted to relevant national and international conferences.

Contributors $A C$ and HS conceptualised the study. MD and GC contributed to the study design. ZL and AC planned the statistical analysis. AC wrote the first draft of the protocol. All authors read and approved the final version.

Funding This work will be supported by the Medical University of Warsaw. The study product will be manufactured by Sequoia Ltd, Poland, free of charge.

Competing interests None declared.

Ethics approval Bioethics Committee of the Medical University of Warsaw, Poland.

Provenance and peer review Not commissioned; externally peer reviewed.

Open Access This is an Open Access article distributed in accordance with the Creative Commons Attribution Non Commercial (CC BY-NC 4.0) license, which permits others to distribute, remix, adapt, build upon this work noncommercially, and license their derivative works on different terms, provided the original work is properly cited and the use is non-commercial. See: http:// creativecommons.org/licenses/by-nc/4.0/

\section{REFERENCES}

1. McCann JC, Ames BN. An overview of evidence for a causal relation between iron deficiency during development and deficits in cognitive or behavioral function. Am J Clin Nutr 2007;85:931-45.

2. Beard J. Iron deficiency alters brain development and functioning. J Nutr 2003;133(Suppl 1):1468S-72S.

3. Lozoff B. Early iron deficiency has brain and behavior effects consistent with dopaminergic dysfunction. J Nutr 2011;141:740S-6S

4. Lozoff B, Beard J, Connor J, et al. Long-lasting neural and behavioral effects of iron deficiency in infancy. Nutr Rev 2006;64 (Suppl):S34-43

5. Wang B, Zhan S, Gong T, et al. Iron therapy for improving psychomotor development and cognitive function in children unde the age of three with iron deficiency anaemia. Cochrane Database Syst Rev 2013;6:CD001444

6. Domellöf M, Braegger C, Campoy C, et al, ESPGHAN Committee on Nutrition. Iron requirements of infants and toddlers. $J$ Pediatr Gastroenterol Nutr 2014:58:119-29.

7. Aggett PJ, Agostoni C, Axelsson I, et al. Do we know enough? A commentary by the ESPGHAN Committee on Nutrition. $J$ Pediatr Gastroenterol Nutr 2002;34:337-45.

8. Beard J. Recent evidence from human and animal studies regarding iron status and infant development. $J$ Nutr 2007;137:524S-30S.

9. Shafir $\mathrm{T}$, Angulo-Barroso $\mathrm{R}$, Jing $\mathrm{Y}$, et al. Iron deficiency and infant motor development. Early Hum Dev 2008;84:479-85.

10. Baker RD, Greer FR, Committee on Nutrition, American Academy of Pediatrics. Diagnosis and prevention of iron deficiency and iron-deficiency anemia in infants and young children (0-3 years of age). Pediatrics 2010;126:1040-105.

11. Szajewska $\mathrm{H}$, Ruszczynski M, Chmielewska A. Effects of iron supplementation in nonanemic pregnant women, infants and young children on the mental performance and psychomotor development of children: a systematic review of randomized controlled trials. $A m \mathrm{~J}$ Clin Nutr 2010;91:1-7.

12. Friel JK, Aziz K, Andrews WL, et al. A double-masked, randomized control trial of iron supplementation in early infancy in healthy term breast-fed infants. J Pediatr 2003;143:582-6.

13. Lind T, Lönnerdal $B$, Stenlund $H$, et al. A community-based randomized controlled trial of iron and zinc supplementation in Indonesian infants: effects on growth and development. Am J Clin Nutr 2004;80:729-36.

14. Moffatt MEK, Longstaffe S, Besant J, et al. Prevention of iron deficiency and psychomotor decline in high risk infants through use of iron fortified infant formula: a randomized clinical trial. J Pediatr 1994:125:527-34

15. Morley R, Abbott R, Fairweather-Tait S, et al. Iron fortified follow on formula from 9 to 18 months improves iron status but not development or growth: a randomized trial. Arch Dis Child 1999;81:247-52.

16. Yalcin SS, Yurdakok K, Acikgoz D, et al. Short-term developmental outcome of iron prophylaxis in infants. Pediatr Int 2000;42:625-30.

17. Pongcharoen T, DiGirolamo AM, Ramakrishnan U, et al. Longterm effects of iron and zinc supplementation during infancy on cognitive function at $9 \mathrm{y}$ of age in northeast Thai children: a follow-up study. Am J Clin Nutr 2011;93:636-43.

18. Abdullah K, Kendzerska $\mathrm{T}$, Shah $\mathrm{P}$, et al. Efficacy of oral iron therapy in improving the developmental outcome of preschool children with nonanaemic iron deficiency: a systematic review. Public Health Nutr 2013;16:1497-506.

19. Domellöf M, Cohen RJ, Dewey KG, et al. Iron supplementation of breast-fed Honduran and Swedish infants from 4 to 9 months of age. $J$ Pediatr 2001;138:679-87.

20. Dewey KG, Domellöf M, Cohen RJ, et al. Iron supplementation affects growth and morbidity of breast-fed infants: results of a randomized trial in Sweden and Honduras. $J$ Nutr 2002;132:3249-55.

21. Mora JO. Iron supplementation: overcoming technical and practical barriers. J Nutr 2002;32:853S-5S.

22. http://toxnet.nlm.nih.gov/cgibin/sis/search/a?dbs+hsdb: @term +@DOCNO+454; (accessed 02 Jul 2015).

23. Hirve S, Bhave S, Bavdekar A, et al. Low dose 'Sprinkles'- an innovative approach to treat iron deficiency anaemia in infants and young children. Indian Pediatr 2007;44:91-100.

24. Bayley N. Bayley scales of infant and toddler development. 3rd edn. San Antonio, TX: Pearson Education, Inc., 2006. (http://www. pearsonclinical.com/childhood/products/100000123/bayley-scalesof-infant-and-toddler-development-third-edition-bayley-iii.html (accesses 02 Jul 2015)

25. Achenbach TM, Rescorla LA. Manual for the ASEBA preschool forms and profiles. Burlington, VT: University of Vermont, Research Center for Children, Youth and Families, 2000

26. Drakesmith $\mathrm{H}$, Prentice AM. Hepcidin and the iron-infection axis. Science 2012;338:768-72.

27. Rishi G, Wallace DF, Subramaniam VN. Hepcidin: regulation of the master iron regulator. Biosci Rep 2015;35:e00192.

28. Berglund $\mathrm{S}$, Lonnerdal $\mathrm{B}$, Westrup $\mathrm{B}$, et al. Effects of iron supplementation on serum hepcidin and serum erythropoietin in low-birthweight infants. Am J Clin Nutr 2011;94:1553-61.

29. http://www.who.int/childgrowth/training/jobaid_weighing_measuring pdf?ua=1 (accessed 15 Jul 2015)

30. http://www.izz.waw.pl/pl/usugi?id=450 (accessed 1 Sep 2015).

31. Armijo-Olivo S, Warren S, Magee D. Intention to treat analysis, compliance, drop-outs and how to deal with missing data in clinical research: a review. Phys Ther Rev 2009;14:36-49.

32. Karlson CW, Rapoff MA. Attrition in randomized controlled trials for pediatric chronic conditions. J Pediatr Psychol 2009;34:782-93. 